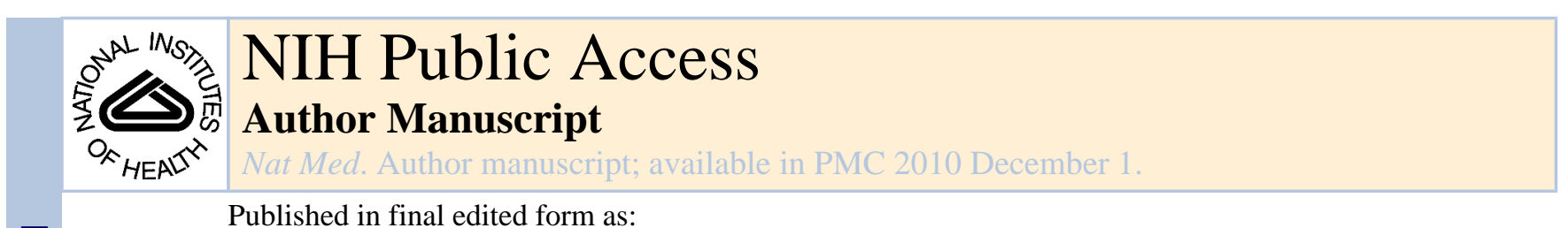

Published in final edited form as:

Nat Med. 2010 June ; 16(6): 641-644. doi:10.1038/nm0610-641.

\title{
Shifting HIFs in osteoarthritis
}

\author{
Matthew Husa, Ru Liu-Bryan, and Robert Terkeltaub \\ The Veterans Affairs Medical Center and University of California-San Diego, San Diego, California, \\ USA
}

Robert Terkeltaub: rterkeltaub@ucsd.edu

\begin{abstract}
There is no cure for osteoarthritis-the most common disease of the joints. By piecing together the molecular events that drive the progression of this debilitating disease, recent studies published in Nature Medicine put hypoxia-inducible factor- $2 \alpha(\mathrm{HIF}-2 \alpha)$ in the driver's seat, opening up new avenues for early detection and treatment (pages 678-686 and 687-693).
\end{abstract}

Osteoarthritis is the most common form of arthritis, and it is an enormous, expensive public health problem. The characteristic features of this chronic, progressive, degenerative disorder of the entire joint include variable inflammation of the synovium - the thin membrane around the joint that secretes the lubricating synovial fluid — and changes in the structure of the bone beneath and bordering the joint and in the protective cushion, called articular cartilage, that allows low-friction movement between the ends of long bones.

Osteoarthritis causes loss of articular cartilage, accompanied by fibrosis of the bone marrow, as well as thickening and other abnormalities of the subchondral bone, the layer of bone just below the cartilage. These processes are linked with the symptoms of joint pain. In addition, new bone growth results in the formation of osteophytes, or bony projections at the edges of the joint. Over years, this process can culminate in marked loss of articular cartilage that results in bone grinding on bone, leading to functional failure of the joint and, ultimately, disability. Although the cause of osteoarthritis is not known, age, biomechanical injury and heredity are primary risk factors.

There are no effective medical therapies to prevent cartilage destruction and the associated bony changes in the joint in osteoarthritis. This situation reflects an insufficient understanding of the molecular mechanisms involved in the condition.

The insight needed to develop disease-modifying osteoarthritis therapeutics now takes a large step forward with two studies in this issue of Nature Medicine. Saito et al. ${ }^{1}$ and Yang et al. ${ }^{2}$ describe a central role for the transcription factor HIF-2 $\alpha$ in disease pathogenesis. These findings, together with other recent papers in Nature Medicine, by Lin et al. ${ }^{3}$ and Echtermeyer et $a l .{ }^{4}$, describing additional molecular players in osteoarthritis, help build a new model for the development of osteoarthritis. According to this paradigm, a stress-induced increase in the activity of HIF- $2 \alpha$, overshadowing the beneficial effects of the closely related HIF- $1 \alpha$, pushes the cartilage-producing cells, or chondrocytes, in the joint toward a more differentiated state known as hypertrophy, which then drives osteoarthritis (Fig. 1).

Competing Financial Interests: The authors declare no competing financial interests. 


\section{Current therapeutic targets}

Chondrocytes are the sole cells populating joint cartilage, and they normally function to maintain, remodel and repair this nonvascularized tissue. These cells must be remarkably hardy, as they subsist on the diffusion of gaseous nutrients through cartilage in a hypoxic environment (that is, $\sim 2-10 \%$ partial pressure of oxygen $\left(\mathrm{pO}_{2}\right)$ relative to $21 \%$ atmospheric $\mathrm{pO}_{2}$ ).

Chondrocytes interpret and respond to biomechanical stressors, including shear, strain and compressive forces, by altering the extracellular matrix around them. These cells balance anabolic (matrix-building) processes, including the synthesis of matrix proteins such as fibrillar type II collagen, and catabolic processes, including proteases that degrade these matrix constituents. Essentially, osteoarthritis reflects an imbalance between matrix anabolic and catabolic processes.

Several remarkable studies have revealed a role in osteoarthritis for increased chondrocyte expression and activation of proteases, including matrix metalloproteinases (MMPs) and aggrecanases of the a disintegrin and metalloproteinase with thrombsopondin motifs (ADAMTS) family. As a result of these studies, ADAMTS5 and MMP-13 are considered to be leading rational targets for a new generation of osteoarthritis therapeutics ${ }^{5}$. However, achieving selective, safe and effective targeting of proteases to suppress osteoarthritis progression remains a challenge ${ }^{5}$, in part due to the needs for drug specificity with respect to possible unintended effects on physiologic functions of other members of the ADAMTS and MMP families.

A number of alternative or complementary approaches to selective protease inhibition also merit consideration. These include methods to minimize loss of chondrocyte viability, thereby helping maintain a critical chondrocyte mass. For example, one target may be chondrocyte apoptosis, which increases in osteoarthritic cartilage. Another approach could be to promote chondrocyte autophagy, given that aging and osteoarthritic cartilages show deficiencies in this process-a cellular mechanism to enhance cell survival by energy-efficient recycling and repair of damaged cell components, including major organelles ${ }^{6}$.

Yet another possible therapeutic target is now suggested by the emerging osteoarthritis model buttressed by the four Nature Medicine studies ${ }^{1-4}$. In this model, a maturation switch turned on in stressed cartilage pushes resting chondrocytes toward hypertrophy, which helps ignite and also drive osteoarthritis ${ }^{1-4}$. This paradigm is not entirely new ${ }^{7-9}$, and it is related to the concept that osteoarthritis is an imbalance between matrix anabolic and catabolic processes. But these studies now provide hard evidence that hypertrophy tips the scales in favor of catabolism in osteoarthritis in vivo ${ }^{1-4,9}$.

\section{A new target: chrondrocyte hypertrophy}

During bone development, mesenchymal stem cells differentiate into chondrocytes or osteoblasts, depending on the environment and signals provided. Differentiation in a vascularized area promotes osteoblast development, whereas differentiation of a mesenchymal stem cell in a poorly vascularized, hypoxic area favors chondrocyte development and cartilage formation.

Chondrocytes at the growth plate - the areas of developing tissue near the end of long bones -undergo exhaustive rounds of spatially ordered proliferation exquisitely controlled by multiple mediators ${ }^{10,11}$ before undergoing hypertrophy. Growth plate chondrocytes build bone by promoting increased vascularity, bone cell ingress and calcification and degrading existing cartilage extracellular matrix to make room for the developing bone. This process, known as 
endochondral ossification, is essential to the elongation of long bones. The hypertrophic chondrocyte at the growth plate is defined not only by its increased diameter, but also by markers including type X collagen, MMP-13 and vascular endothelial growth factor (VEGF), as well as a decrease in type II collagen ${ }^{1,2,7-9}$.

Chondrocyte hypertophy has also been observed to occur in osteoarthritic cartilage, but the importance of such hypertrophy in osteoarthritis and the mechanisms by which it develops have been debated for nearly two years. In articular cartilage, hypertrophic chondrocytes express many of the same markers of hypertrophy seen in growth plate. Because in the growth plate hypertrophy occurs in one of the later stages of endochondral ossification, some researchers have suggested that hypertrophy is primarily a late differentiation event of osteoarthritic chondrocytes.

The new studies, however, support the conclusion that articular chondrocyte hypertrophy is an earlier event than previously believed ${ }^{1-4,9}$. The studies suggest that such hypertrophy may reflect a frustrated, and ultimately futile, process to repair stressed articular cartilage ${ }^{1-4,9}$. The articular chondrocyte hypertrophy partially recapitulates normal endochondral development in that the degrading and remodeling of the extracellular matrix promotes pathologic cartilage calcification, which is observed both in osteoarthritis and in age-related chondrocalcinosis.

\section{Homing in on the HIFs}

What is the mechanism that drives this pathologic chondrocyte hypertrophy? Biomechanical stress, altered matrix-cell communication and inflammatory mediators seem central to the process. Hypertrophy in cultured chondrocytes can also occur as a result of cyclic tensile strain ${ }^{12}$ and of the autocrine and paracrine effects of collagen II peptides and of ligands of a variety of innate immune, inflammatory cytokine and patterning receptors ${ }^{7,13}$. Chondrocyte differentiation and function is also affected by the diffusion into cartilage of soluble inflammatory mediators from subchondral osteoblasts ${ }^{14}$, synovial lining cells and other cells in the joint. Notably, the inflammation-induced articular chondrocyte hypertrophy program seems to occur without antecedent repetitive rounds of proliferation, unlike the case in the growth plate ${ }^{8}$. In essence, articular chondrocyte hypertrophy in osteoarthritis does not seem to be a physiological process.

Together, the independent Nature Medicine publications by Saito et al. ${ }^{1}$ and Yang et al. ${ }^{2}$ reveal a blueprint by which stresses including inflammation and biomechanical injury converge on chondrocyte hypertrophy in osteoarthritis (Fig. 1). This convergence occurs through the activity of HIF- $\alpha$, which transduces cell responses to changes in oxygen concentration and is a member of a family of hypoxic response transcription factors.

The studies reveal that HIF-2 $\alpha$ expression is induced by multiple proinflammatory cytokines produced in large part by chondrocytes, including TNF- $\alpha$ and interleukin-1 $\beta$ (IL-1 $\beta$ ), but not anti-inflammatory cytokines such as IL-4 and IL-10. The striking effects of IL- $1 \beta$ on HIF- $2 \alpha$ expression are transduced by c-Jun N-terminal kinase (JNK) and nuclear factor- $\mathrm{\kappa B}$ (NF- $\mathrm{KB}$ ) signaling. The v-rel reticuloendotheliosis viral oncogene homolog A (RelA, or NF- $\mathrm{BB}$ p65), a core molecule in NF- $\kappa \mathrm{B}$ transcriptional signaling, has a major role in this signaling mechanism ${ }^{1}$. The finding of Re1A involvement in expression of HIF- $2 \alpha$ is noteworthy partly because NF- $\kappa \mathrm{B}$ signaling is also known to transduce biomechanical injury in chondrocytes ${ }^{15}$.

Saito et al. ${ }^{1}$ and Yang et al. ${ }^{2}$ also show that increased HIF-2 $\alpha$ activity is sufficient to promote the cartilage hypertrophy gene expression program in vitro, including induction of multiple proteases that degrade cartilage extracellular matrix. Moreover, HIF- $2 \alpha$ can promote expression of the aggrecanase ADAMTS4, the inflammation mediator prostaglandin- 
endoperoxide synthase-22 and nitric oxide synthase 2-which acts, via nitric oxide generation, to decrease chondrocyte proteoglycans synthesis and increase chondrocyte apoptosis.

HIF-2 $\alpha$ expression is increased in the early and progressive stages of disease in human osteoarthritic cartilages, as well as in the cartilages of mouse models of knee osteoarthritis induced by surgery and in association with aging ${ }^{1,2}$. Furthermore, increasing HIF- $2 \alpha$ expression specifically in the joint cartilage, using either transgenic methodology or transduction using adenoviral HIF-2 $\alpha$, induces osteoarthritis ${ }^{1,2}$. Conversely, HIF- $2 \alpha$ deficiency protects against cartilage destruction and osteophyte formation in mouse models of osteoarthritis in which the disease develops weeks to months after the disruption of articular connective tissues to promote cartilage biomechanical injury ${ }^{1,2}$.

HIF-2 $\alpha$ modulates endochondral ossification. Moreover, the HIF-2 $\alpha-$ deficient mice had no gross abnormalities of major organs, and HIF- $2 \alpha$-haploinsufficient mice showed only mild dwarfism under conditions in which osteoarthritis progression was markedly reduced via the HIF- $2 \alpha$ haploinsufficiency. These results leave open the hope that it might be possible to selectively target HIF-2 $\alpha$ as a treatment for human osteoarthritis without major side effects.

\section{A delicate balance}

A stunning aspect of this HIF-mediated model for osteoarthritis is that it seems to involve a shift in the balance between HIF-1 $\alpha$ and HIF- $2 \alpha$ (Fig. 1). There are three known types of HIF$\alpha$, although HIF- $3 \alpha$ is not robustly expressed in chondrocytes ${ }^{1}$. Each HIF- $\alpha$ subunit has the potential to heterodimerize with several HIF- $\beta$ subunits, which are called aryl hydrocarbon receptor nuclear translocators (ARNTs). More specifically, they are ARNT, ARNT2, ARNTlike (ARNTL) and ARNTL2. Under hypoxic conditions, HIF heterodimers translocate to the nuclei of cells, including chondrocytes, and bind gene promoters bearing consensus hypoxia response elements (HREs), activating the expression of the genes. In this manner, hypoxia promotes transcription of genes involved in energy production and use and angiogenesis, allowing multiple benign and malignant cells to manage stress and survive in challenging environments.

HIF- $1 \alpha$ and HIF- $2 \alpha$ are approximately 50\% homologous at the amino acid level, yet there are remarkable differences in their expression and function in cartilage, as well as in other tissues. For example, HIF-1 $\alpha$ is expressed mainly in hypoxic, poorly vascularized sites, whereas both hypoxic and well-vascularized tissues express HIF- $2 \alpha$. A clear new finding from the studies of Saito et al. ${ }^{1}$ and Yang et al. ${ }^{2}$ is that the functional differences between HIF-1 $\alpha$ and HIF-2 $\alpha$ in articular chondrocytes in the pathogenic state of osteoarthritis seem to mirror distinct roles of these HIFs in the growth plate under physiological conditions. HIF- $1 \alpha$ is central in the early stages of cartilage formation. HIF- $1 \alpha$ also seems to function in maintaining baseline chondrocyte differentiation and production of a normal, collagen II-rich extracellular matrix under hypoxic conditions ${ }^{16}$. Moreover, HIF- $1 \alpha$ promotes chondrocyte autophagy, and, importantly, this beneficial effect is antagonized by HIF-2 $\alpha$ in cultured chondrocytes ${ }^{17}$ (Fig. 1). HIF- $2 \alpha$ is expressed more in well-differentiated chrondocytes than in nascent cells, and many HIF-2 $\alpha$ functions, including the promotion of chondrocyte hypertrophy ${ }^{1}$, are not subject to loss by oxygen-dependent prolyl hydroxylation.

The expression and activity of HIFs are regulated by enzymes known as prolyl hydroxylases. These enzymes become active in the presence of oxygen and target the $\alpha$ subunits of HIFs for prolyl hydroxylation, a chemical modification that marks proteins for subsequent ubiquitination and proteasomal degradation. In the absence of oxygen, prolyl hydroxylases are deactivated, and HIFs are stabilized. An intriguing question that arises from these studies is whether the pathogenesis of osteoarthritis also involves regulation of prolyl hydroxylases that differentially act on HIF- $1 \alpha$ and HIF- $2 \alpha$ in cartilage during disease progression. 
Another key consideration is the role that the differential regulation and functions of ARNTs have in chondrocyte hypertrophy and osteoarthritis. All ARNT $\beta$-subunit members can physically associate with HIF- $2 \alpha$, but ARNTL shows the highest binding affinity, and in cartilage it co-localizes more frequently with HIF- $2 \alpha$ than with HIF- $1 \alpha^{1}$. A striking finding by Saito et al. ${ }^{1}$ was that HIF- $2 \alpha$ and ARNTL are the most potent set of partners for inducing several elements of the chondrocyte hypertrophy gene expression program. One possibility is that ARNTL may turn out to be more 'druggable' than HIF-2 $\alpha$ for osteoarthritis.

\section{How HIF-2 $\alpha$ does it}

HIF-2 $\alpha$ directly stimulates transcription of some, but not all, major players involved in the pathogenetic transformation of an osteoarthritic chondrocyte ${ }^{1,2}$. Nevertheless, HIF- $2 \alpha$ can be conceptually planted in a central region of a disease paradigm focused on chondrocyte hypertrophy that incorporates all of the major recent findings (Fig. 1).

Specifically, HIF-2 $\alpha$ induces chondrocyte expression of Indian hedgehog (IHH) ${ }^{1}$, the major hedgehog family ligand in cartilage. IHH signals through the Patched-1 (PTCH1) receptor and glioma-associated oncogene homolog (GLI) family of transcription factors to promote chondrocyte hypertophy both in skeletal development and in osteoarthritis ${ }^{3}$. Hedgehog signaling has previously been implicated in the response to biomechanical stress in chondrocytes. Lin et al. ${ }^{3}$ discovered increased hedgehog signaling in human osteoarthritis and that deficiencies and increases in IHH signaling respectively suppress and promote the development of articular chondrocyte hypertrophy and experimental osteoarthritis in genetically modified mice.

HIF- $2 \alpha$ also can induce runt-related transcription factor-2 (RUNX2) expression in vitro. RUNX2 is not only induced by biomechanical injury in chondrocytes, but also is a mediator of chondrocyte hypertrophy and of MMP-13 expression just weeks into the course of experimental, mouse knee instability-induced osteoarthritis ${ }^{9}$. Experiments have suggested that RUNX2 is involved in the modulation of osteoarthritis by IHH, in part by regulating expression of the protease ADAMTS5 (ref. 3 ), and ADAMTS5 activity plays a key part in instabilityinduced knee osteoarthritis in mice ${ }^{5}$. However, RUNX2 does not seem essential for HIF-2 $\alpha$ to promote osteoarthritis. Also, HIF-2 $\alpha$ (like IL-1 $\beta$ ) does not induce transcription of ADAMTS5 (refs. 1,2) (Fig. 1). Instead, IL-1 $\beta$ and certain other inflammatory mediators promote activation of latent ADAMTS-5, as does hypertrophic differentiation in chondrocytes.

These collective findings are consistent with the observations by Echtermeyer et al. ${ }^{4}$ that hypertrophic chondrocytes express the transmembrane (type I) heparan sulfate proteoglycan syndecan-4. Expression of syndecan-4 increases in osteoarthritis cartilage, promotes disease progression and stimulates ADAMTS5 activation. Interestingly, syndecan-4 stimulates ADAMTS5 activation directly and also indirectly by promoting expression of MMP-3, which not only acts on ADAMTS5 but also degrades certain cartilage extracellular matrix constituents ${ }^{4}$.

Hence, synergy between different pathways, some stimulated by HIF- $2 \alpha$ and some by other means, is a core feature of osteoarthritis chondrocyte biology (Fig. 1). HIF-2 $\alpha$ directly promotes expression of multiple MMP family members and chondrocyte hypertrophy without altering ADAMTS5 expression or activity—but, ultimately, multiple pathways involved in chondrocyte hypertrophy converge on ADAMTS5 activity. Specifically, RUNX2, syndecan-4 and IHH provide complementary or independent means to promote cartilage matrix degradation by ADAMTS5 in hypertrophic chondrocytes. This aspect of the model is important, because some therapies in development have focused on ADAMTS5, but other pathways may offer new means to target ADAMTS5. 


\section{More questions}

This chondrocyte-centered model of osteoarthritis progression is incomplete, even at the level of the chondrocyte. Oxidative stress and the aging process are among many physiogical players that affect chondrocyte function that are not included in this model. Aging has been linked to decreased HIF- $1 \alpha$ activity in tissues other than cartilage. Conversely, HIF- $1 \alpha$ activity promotes longevity in Caenorhabditis elegans, one aspect of the complex relationship between HIF- $1 \alpha$ and longevity ${ }^{18}$. In addition, the systems biology of chondrocyte hypertrophy and potential regulatory effects of chondrocyte microRNAs ${ }^{19}$ on HIF and ARNT expression merit further investigation. Consideration must also be given to paracrine effects of osteoarthritic subchondral osteoblasts, now recognized to promote chondrocyte hypertrophy ${ }^{14}$, as well as to altered $\beta$-catenin activity and the effects of transglutaminase-2 (ref. 8 ).

Other central events in chondrocyte biology thought to have a role in osteoarthritis include impaired signaling of the chondrocyte growth factors insulin-like growth factor-I (IGF-I), transforming growth factor- $\beta$ (a constitutive inhibitor of chondrocyte maturation to hypertrophy) and fibroblast growth factor-2 (FGF-2) (a constitutive suppressor of ADAMTS5

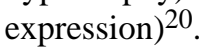

\section{Prospects for therapy}

Selective, rationally designed biologic drugs that clearly slow joint destruction are in clinical use for some diseases of the joint, including rheumatoid arthritis. Might the Nature Medicine studies focused on here ${ }^{1-4}$ be translated into safe and effective therapies for osteoarthritis? Before that can happen, research must close large gaps that still remain in understanding the pathophysiology. Specifically, there is a need for broadly available biomarkers and advanced imaging modalities that better predict and identify the development of osteoarthritis.

Such markers might be used to clinically recognize the onset of chondrocyte hypertrophy and catabolism before loss of cartilage progresses to the point where it is clinically identifiable, or beyond a 'point of no return'. In this light, although HIF- $2 \alpha$ clearly plays a part in triggering the disease, the protein was downregulated in late stages of osteoarthritic lesions ${ }^{1,2}$. This finding argues that the time window for using drugs that inhibit HIF-2 $\alpha$ in the treatment of symptomatic osteoarthritis may be narrow. Moreover, the safety margin for potential HIF- $2 \alpha$ antagonists may be limited, as studies have suggested that HIF- $2 \alpha$ has physiological protective effects against neuronal oxidative stress and normoxic oxidative neuronal death ${ }^{21}$. But the good news is that cartilage HIF- $2 \alpha$ and some of the genes it induces could potentially be tapped as early biomarkers of an 'unhealthy chondrocyte' that ignites and accelerates osteoarthritis.

Increasing chondrocyte HIF-1 $\alpha$ expression may be an alternative therapeutic strategy in osteoarthritis. For example, there is evidence suggesting that exercise, and certain small molecules, including the vasodilator hydralazine, may reverse the age-related decline in neovascularization by increasing HIF- $1 \alpha$-mediated signaling ${ }^{22}$. In addition, the findings of Echtermeyer $\mathrm{et} \mathrm{al} .{ }^{4}$ that intra-articular injection of a syndecan-4-specific antibody reduces proteoglycan loss in osteoarthritic knee cartilage ${ }^{4}$ suggests potential value of approaches that target syndecan-4.

In addition, promoter structure-function studies by Saito et al. ${ }^{1}$ of several genes in the chondrocyte hypertrophy program have identified core responsive elements in certain HREs that alter transactivation by HIF- $2 \alpha$ and the HIF-2 $\alpha$-ARNTL pair. This work has uncovered an osteoarthritis disease susceptibility single nucleotide polymorphism in the in HIF2A gene in a small Japanese cohort. 
The genetics of HIF-2 $\alpha$ and other players in chondrocyte hypertrophy are likely to become fertile areas of investigation in osteoarthritis and potentially useful for preventive and medical treatment strategies involving personalized genomics. Lastly, an improved understanding of how chondrocytes undergo hypertrophy should pay relatively early dividends for improving cell replacement and engineering therapies to repair cartilage defects in vivo.

\section{Acknowledgments}

Supported by the VA Research Service and US National Institutes of Health grants PAGO7996, AR1067966, 5T32AR007608.

\section{References}

1. Saito T, et al. Nat Med 2010;16:678-686. [PubMed: 20495570]

2. Yang S, et al. Nat Med 2010;16:687-693. [PubMed: 20495569]

3. Lin AC, et al. Nat Med 2009;15:1421-1425. [PubMed: 19915594]

4. Echtermeyer F, et al. Nat Med 2009;15:1072-1076. [PubMed: 19684582]

5. Flannery CR. Curr Drug Targets 2010;11:614-619. [PubMed: 20199389]

6. Caramés B, et al. Arthritis Rheum 2010;62:791-801. [PubMed: 20187128]

7. Cecil DL, et al. J Immunol 2009;182:5024-5031. [PubMed: 19342682]

8. Johnson KA, Terkeltaub RA. J Biol Chem 2005;280:15004-15012. [PubMed: 15691824]

9. Kamekura S, et al. Arthritis Rheum 2006;54:2462-2470. [PubMed: 16868966]

10. Akiyama H, et al. Genes Dev 2004;18:1072-1087. [PubMed: 15132997]

11. Vega RB, et al. Cell 2004;119:555-566. [PubMed: 15537544]

12. Wong M, et al. Bone 2003;33:685-693. [PubMed: 14555274]

13. Liu-Bryan R, Terkeltaub R. Arthritis Rheum. in the press.

14. Prasadam I, et al. Arthritis Rheum 2010;62:1349-1360. [PubMed: 20155832]

15. Nam J, et al. PLoS One 2009;4:e5262. [PubMed: 19370157]

16. Lafont JE, et al. Arthritis Rheum 2007;56:3297-3306. [PubMed: 17907154]

17. Bohensky J, et al. Pediatr Nephrol 2010;25:633-642. [PubMed: 19830459]

18. Zhang Y, et al. PLoS One 2009;4:e6348. [PubMed: 19633713]

19. Miyaki S, et al. Arthritis Rheum 2009;60:2723-2730. [PubMed: 19714579]

20. Chia SL, et al. Arthritis Rheum 2009;60:2019-2027. [PubMed: 19565481]

21. Siddiq A, et al. J Neurosci 2009;29:8828-8838. [PubMed: 19587290]

22. Hoenig MR, et al. Curr Mol Med 2008;8:754-767. [PubMed: 19075673] 


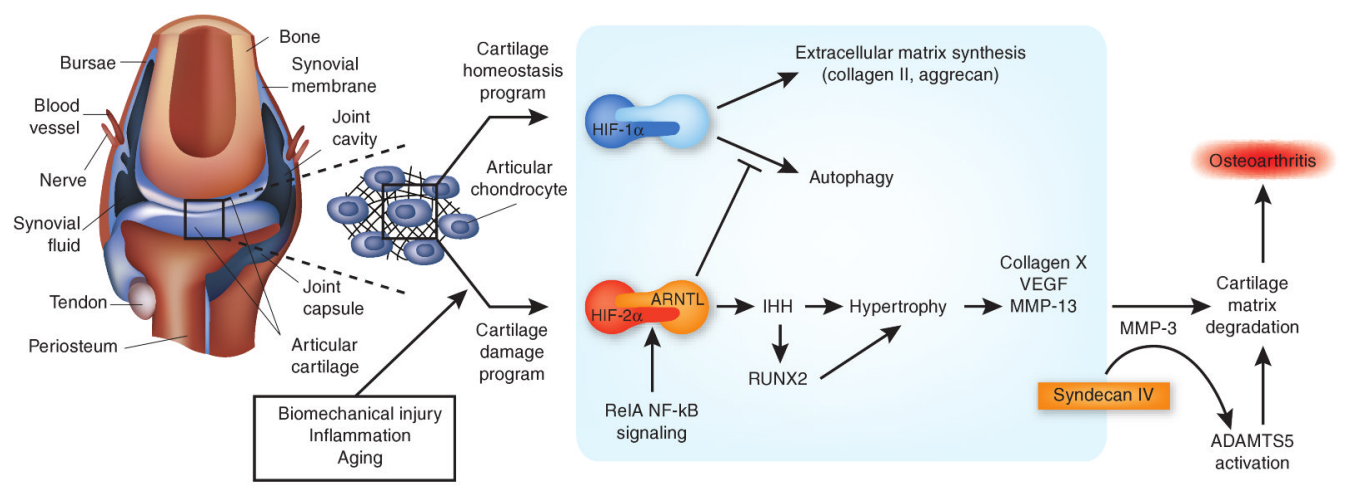

Figure 1.

HIF- $1 \alpha$ and HIF- $2 \alpha$ have different functions in the cartilage. In the articular chondrocytes in the synovial joint, HIF- $1 \alpha$ promotes homeostatic pathways, and HIF-2 $\alpha$ promotes degradative pathways that foster osteoarthritis. The articular cartilage resides in hypoxic, avascular conditions within the synovial joint (left). Chondrocytes, cells of the articular cartilage, are affected by various forms of stress (biomechanical, inflammatory and aging), as well as the loss of synovial fluid boundary lubricants and the increase of certain factors released from subchondral bone and synovium. Four studies published in Nature Medicine have provided details for the emerging chondrocyte-centered osteoarthritis model depicted here ${ }^{1-4}$. Within the normal, unstressed chrondocyte (HIF-1 $\alpha$ pathway), the hypoxic response transcription factor HIF-1 $\alpha$ supports normal cartilage extracellular matrix synthesis and chondrocyte differentiation and promotes autophagy_all central activities in articular cartilage homeostasis. These effects of HIF- $1 \alpha$ are antagonized by the closely related HIF- $2 \alpha$ (HIF-2 $\alpha$ pathway). HIF-2 $\alpha$ promotes chondrocyte hypertrophy, a terminal differentiation state characterized by a unique gene expression program, including type X collagen and the type II collagen-degrading protease MMP-13. This switch to hypertrophy seems to be a relatively early signal to ignite and drive osteoarthritis in stressed cartilage. The preferential heterodimerization of HIF-2 $\alpha$ with ARNTL creates the most potent set of partners for inducing chondrocyte hypertrophy. HIF-2 $\alpha$ promotes hypertrophy, and complementary mechanisms (via IHH and RUNX2) stimulate increased expression of the major matrix-degrading protease ADAMTS5. The transmembrane heparan sulfate proteoglycan syndecan-4 (acting in part by inducing MMP-3 expression) stimulates activation of ADAMTS5 in hypertrophic chondrocytes. Inhibitors of ADAMTS5 and MMP-13 are already in clinical development. The model depicted here opens the door to additional, compelling strategies for potential disease modification in human osteoarthritis, such as suppressing the activity of HIF-2 $\alpha$, inhibiting the development of articular chondrocyte hypertrophy or targeting syndecan-4. 